\title{
Editorial
}

\section{Recent Advancements in Signal Processing and Machine Learning}

\author{
Gelan Yang, ${ }^{1}$ Su-Qun Cao, ${ }^{2}$ and Yue $\mathrm{Wu}^{3}$ \\ ${ }^{1}$ College of Information Science and Engineering, Hunan City University, Yiyang 413000, China \\ ${ }^{2}$ Faculty of Mechanical Engineering, Huaiyin Institute of Technology, Huaian 223003, China \\ ${ }^{3}$ Department of Electrical and Computer Engineering, Tufts University, Medford, MA 02155, USA
}

Correspondence should be addressed to Gelan Yang; glyang@mail.ustc.edu.cn

Received 26 March 2014; Accepted 26 March 2014; Published 14 April 2014

Copyright (C) 2014 Gelan Yang et al. This is an open access article distributed under the Creative Commons Attribution License, which permits unrestricted use, distribution, and reproduction in any medium, provided the original work is properly cited.

Recent advances of consumer electronics, like iphone 5s, google glasses, Xbox Kinect, and so forth, bring people revolutionary experiences of human-machine interactions. Behind these innovations are the successes of various recent cutting-edge technologies, including voice recognition, object recognition, and motion recognition. Though many of these technologies are still far from perfect, these examples demonstrate the usefulness and importance of signal processing and machine learning (SPML) research.

SPML, however, clearly goes far beyond these wellknown recognition technologies and roots deeply in many aspects of academic research and industrial development. Indeed, wherever digital sensors require signal processing, and wherever decision-making problems can be formed in a machine learning manner. Moreover, SPML is also deeply involved: on one hand, ML could take digital signals as raw features to learn rules; on the other hand, many statistical SP techniques are essentially ML solutions. For example, wavelet transform proposed for SP now has been widely used as a preprocessing for many machine learning applications, while probabilistic graphic models often used in an expert system now show their potentials in image segmentation and recognition.

For these reasons, the aim of this special issue is to consider the recent advancements in SPML together. In total, this special issue contains thirty-four papers studying various SPML problems including image registration, pattern recognition, texture analysis, surveillance, biometrics, humanmachine interface, image/video compression/encryption, image enhancement, and face recognition. A summarized description of these papers is given below.
"Strategies for exploiting independent cloud implementations of biometric experts in multibiometric scenarios" by $\mathrm{P}$. Peer et al. presents an analysis of different strategies for combining independent cloud implementations of biometric experts into a multibiometric recognition system. Analysis results suggest that fixed fusion rules combining single expert systems at the matching score level are the most suitable for the studied task as they provide a good balance among expected performance gains and other important factors.

"Linear chromatic adaptation transform based on Delaunay triangulation" by R. Kreslin et al. suggests a method using Delaunay triangulation and linear transformations in order to transform the input image. It relies on the color values of the patches of the Macbeth color checker captured under the same illuminant as the input image must be known. Objective evaluation showed that the proposed method outperforms existing CAT methods by more than $21 \%$, which performs statistically significantly better than that of other existing methods.

"Language recognition using latent dynamic conditional random field model with phonological features" by S. Boonsuk et al. proposes an acoustic SLR system based on the latentdynamic conditional random field (LDCRF) model using phonological features (PFs). Evaluated on the NIST LRE 2007 corpus, the proposed method showed an improvement over the baseline systems. Additionally, it showed comparable result with the acoustic system based on $i$-vector. This research demonstrates that utilizing the $\mathrm{PF}$ attributes as a mean to integrate linguistic information with the acoustic approach can enhance the performance. 
"Human skeleton model based dynamic features for walking speed invariant gait recognition" by J. Kovač et al. proposes a skeleton model based gait recognition system focusing on modelling gait dynamics and eliminating the influence of subject appearance on recognition. Furthermore, this paper tackles the problem of walking speed variation and proposes space transformation and feature fusion that mitigates its influence on recognition performance.

"Efficient LED-SAC sparse estimator using fast sequential adaptive coordinate-wise optimization (LED-2SAC)" by $\mathrm{T}$. Yousefi Rezaii et al. presents a novel method benefits from the sparsity of the signal both in the optimization criterion (LED) and its solution path, denoted by Sparse SAC (2SAC). The new reconstruction method is consequently more efficient and considerably faster compared to the LED-SAC algorithm, in terms of adaptability and convergence rate with a much lower computational complexity.

"A joint learning approach to face detection in wavelet compressed domain" by S.-H. Huang et al. carries out a novel face detection system working directly in the wavelet compressed domain. This methodology involves a feature space warping process, a paired feature learning scheme, an ID3-like joint feature plane quantization method, and a weak Bayesian classifier. Experimental results on the benchmarking face datasets showed that the proposed face detection system working in the compressed domain achieves similar accuracy to that of Viola and Jones' face detector.

"Improvement and simulation of an autonomous time synchronization algorithm for a layered satellite constellation" by F. Huang et al. investigates the autonomous time synchronization algorithm that corresponds to the layered constellation structure, analyzes the main error of the time synchronization algorithm, and proposes methods to improve the characteristics of satellite movement in the constellation. Its simulation results show that in a condition with simulation errors, the time synchronization precision of this improved algorithm can be controlled within $5 \mathrm{~ns}$ and used in high-precision autonomous time synchronization between layered satellite constellations.

"The new mathematical model of motion compensation for stepped-frequency radar signal" by Y. Lin et al. presents a novel mathematical method to estimate target speed for the stepped-frequency radar. Its numeric simulation results confirm that this new method is effective and predominant in terms of much higher estimation accuracy in a low SNR and much larger estimation range of target speed.

"Human walking pattern recognition based on KPCA and SVM with ground reflex pressure signal" by Z. Peng et al. investigates an algorithm based on the ground reflex pressure (GRF) signal obtained from a pair of sensing shoes for human walking pattern recognition. Experimental results showed that algorithm fusing SVM and KPCA had better recognition performance.

"Application of global optimization methods for feature selection and machine learning" by $\mathrm{S}$. Wu et al. proposes a novel immune clonal genetic algorithm for the feature selection problem. The proposed algorithm largely simplifies the feature selection process without trading off its effectiveness.
It shows higher classification accuracy than compared feature selection algorithms.

"An initial value calibration method for the wheel force transducer based on memetic optimization framework" by G. Lin et al. proposes an automatic solution without additional calibration equipment or manual operation. In this method, a vehicle with the wheel force transducer (WFT) is driven on a flat road with a constant speed. A real WFT data is used to verify the proposed method and result shows that it is superior to traditional solutions and can improve the measurement accuracy effectively.

"A routing algorithm for WiFi-based wireless sensor network and the application in automatic meter reading" by $\mathrm{L}$. $\mathrm{Li}$ et al. introduces a new architecture of WiFi-based wireless sensor network, which is suitable for the next generation AMR system. It also proposes a new improved routing algorithm called energy saving-based hybrid wireless mesh protocol (E-HWMP).

"An efficient web usage mining approach using chaos optimization and particle swarm optimization algorithm based on optimal feedback model" by L. Dai et al. proposes an efficient particle swarm chaos optimization mining algorithm. It uses a user feedback model to provide a listing of best-matching webpages for user. Its test results show that this approach significantly outperforms other algorithms in the aspects of response time, execution time, precision, and recall.

"Video shot boundary recognition based on adaptive locality preserving projections" by Y. Xiao et al. introduces a novel video shot boundary recognition method. It firstly defines the discriminating similarity with mode prior probabilities and an adaptive neighborhood selection strategy and then uses an optimized multiple kernel support vector machine to classify video frames into boundary and nonboundary frames.

"A virtual channels scheduling algorithm with broad applicability based on movable boundary" by Y. Tian et al. presents a novel algorithm for virtual channel scheduling based on movable boundary. It divides slots into synchronous ones and asynchronous ones, reduces the scheduling delay, and improves the channel utilization ratio. The proposed method outperforms the DSA algorithm, when the time delay and applicability scope are concerned. In addition, the algorithm is suitable for the scheduling of diverse data sources.

"Comprehensive models for evaluating rock mass stability based on statistical comparisons of multiple classifiers" by L. Dong and X. Li demonstrates the applicability and feasibility of RF, SVM, Bayes (NBC), Fisher, LR, and NN classification models to evaluate the rockmass stability of slope. Results show that the established RF, SVM, Bayes, Fisher, LR, and NN classification models can evaluate the slope status with a high accuracy.

"Unsupervised optimal discriminant vector based feature selection method" by S.-Q. Cao and J. H. Manton proposes an efficient unsupervised feature selection method based on unsupervised optimal discriminant vector. It aims to find important features without using class labels. Two experiments on Wine dataset and fault diagnosis demonstrate that the proposed method is able to find important features and is a reliable and efficient feature selection methodology compared to SUD and Relief-F methods. 
"Penalized maximum likelihood algorithm for positron emission tomography by using anisotropic median-diffusion" by Q. He and L. Huang presents an approach to improving the quality of positron emission tomography images. By fusing an anisotropic median-diffusion filter to maximum-likelihood expectation-maximization algorithm, testing results demonstrate high-quality image reconstruction and denoising with better edge preserving capacities.

"A novel fusion method by static and moving facial capture" by S. Liu et al. introduces a fusion facial detection method in moving environment in the field of face recognition. Experimental results show that this method has better robustness and accuracy.

"Lower power design for UHF RF CMOS circuits based on the power consumption acuity" by N. Xiang-jie and L. Hua conveys a lower power design for UHF RF CMOS circuit based on the power consumption acuity due to the excessive energy consumption of UHF tag. The simulation results show that the leakage power of rectifier in this method is obviously less than the conventional rectifier. The proposed design method is suitable for various industrial productions, public management, and daily life use.

"Accurate counting bloom filters for large-scale data processing" by W. Li et al. proposes a multilevel optimization approach to build an accurate counting bloom filter (ACBF) for reducing the false positive probability. Experiments on realistic datasets show that $\mathrm{ACBF}$ can greatly reduce the false positive probability as well as the map outputs. Meanwhile, compared to the classic solution, this method also improves the join execution times by $20 \%$.

"A heuristic feature selection approach for text categorization by using chaos optimization and genetic algorithm" by $\mathrm{H}$. Chen et al. proposes a novel text categorization algorithm called chaos genetic feature selection optimization. Experimental results show that the proposed algorithm effectively simplifies the feature selection process which can also obtain higher classification accuracy with a smaller feature set.

"A simple and high performing rate control initialization method for H.264 AVC coding based on motion vector map and spatial complexity at low bitrate" by Y. Wu and S.-W. Ko describes a simple and high performance initial QP determining method based on motion vector map. Simulation results indicate that this algorithm outperforms conventional methods under many objective and subjective criteria.

"Subband adaptive filtering with $l_{1}$-norm constraint for sparse system identification" by Y.-S. Choi introduces a normalized subband adaptive filter NSAF integrating a new weighted $l_{1}$-norm constraint. Numerical results prove that the $l_{1}$-norm regularized NSAFs outperform the classical NSAF solutions, especially for identifying a sparse system.

"Image encryption using the chaotic Josephus matrix" by G. Yang et al. introduces a new image encryption solution using the chaotic Josephus matrix. It extends the conventional Josephus traversing to a matrix form and proposes a treatment to improve the randomness of this matrix by mixing chaotic maps. The proposed CJPM is parametric and is uniquely dependent on the set of parameters, which is sufficiently large to provide a secure size of key space. Simulation results demonstrate that an encrypted image of using this method is very random-like from the perspective of human visual inspection.

"Active semisupervised clustering algorithm with label propagation for imbalanced and multidensity datasets" by M. Leng et al. provides an active semisupervised clustering algorithm based on active data selection and semisupervised clustering algorithm on multidensity and imbalanced datasets. Testing results show that the proposed semisupervised clustering has higher accuracy and stable performance compared to other clustering and semisupervised clustering algorithms, especially in case of the datasets are multidensity and imbalanced.

"A novel machine learning strategy based on twodimensional numerical models in financial engineering" by Q. Xu puts forward a two-dimensional numerical model for machine learning to simulate major US stock market index, which uses a nonlinear implicit finite-difference method to find numerical solutions of the two-dimensional simulation model. For the purpose of better prediction of the future trend of the index, experimental results show that the proposed algorithm reduces the prediction error and improve forecasting precision.

"Analysis and denoising of hyperspectral remote sensing image in the curvelet domain" by $\mathrm{D}$. $\mathrm{Xu}$ et al. provides a new denoising algorithm based on the characteristics of hyperspectral remote sensing image (HRSI) in the curvelet domain. The detailed subband images in the same scale and same direction from different wavelengths of HRSI are stacked to obtain new 3D datacubes of the curvelet domain. The multiple linear regression method is also introduced. The simulated data experimental results show that the proposed algorithm is superior to the compared algorithms in the reference in terms of SNR.

"Efficient interaction recognition through positive action representation" by $\mathrm{T}$. $\mathrm{Hu}$ et al. proposes a novel approach to decompose two-person interaction into a positive action and a negative action for more efficient behavior recognition. In this way, interaction recognition can be simplified to positive action-based recognition. Also, they created a new dataset with six types of complex human interaction. Experimental results showed that the proposed recognition technique is more accurate than the traditional method, shortens the sample training time, and hence achieves comprehensive superiority.

"Tensorial kernel principal component analysis for action recognition" by C. Liu et al. proposes a novel tensorial kernel principal component analysis (TKPCA) for feature extraction from action objects, which extends the conventional principal component analysis (PCA) in two perspectives: working directly with multidimensional data (tensors) in their native state and generalizing an existing linear technique to its nonlinear version by applying the kernel trick. Experiments with real action datasets show that the proposed method is insensitive to both noise and occlusion and performs well compared with state-of-the-art algorithms.

"Low-Complexity compression algorithm for hyperspectral images based on distributed source coding" by Y. Nian et al. proposes distributed compression algorithm for realizing 
both lossless and lossy compression. It is implemented by performing scalar quantization strategy on the original hyperspectral images followed by distributed lossless compression. Multilinear regression model is introduced for improving the quality of side information. Experimental results show that the compression performance of the proposed algorithm is competitive with that of state-of-the-art compression algorithms for hyperspectral images.

"Model for recognizing key factors and applications thereof to engineering" by B. Shi and G. Chi presents a model for recognizing key factors while using collinearity diagnostics for deleting factors of repeated information and logistic regression for selecting factors. Experimental results from 2044 observations in finical engineering show that the 13 indicators are recognized as key factors to distinguish the good customers from the bad customers and thus demonstrate the effectiveness of the proposed method.

"Application of fuzzy set theory to quantitative analysis of correctness of the mathematical model based on the ADI method during solidification" by X. Niu et al. proposes a model based on the equivalent specific heat method and the ADI method aiming at improving the computational efficiency. Experimental results show that for a thick-walled position, the time step influences the simulation results of the temperature field and the number of casting meshes has little influence on the simulation results of temperature field, while for a thin-walled position a larger influence exists.

"Minimum error thresholding segmentation algorithm based on 3D grayscale histogram" by J. Liu et al. proposes a novel algorithm called three-dimensional minimum error thresholding (3D-MET) according to the relative entropy theory. The 3D histogram is obtained by combining raw information of pixel intensity distribution and relevant information of neighboring pixels within an image. Experimental results indicate that the proposed approach provides superior segmentation performance compared to other methods for gray image segmentation.

\section{Acknowledgments}

Thanks are due to the authors of the special issue for their contributions and thanks to the reviewers for their valuable comments on the submissions. Gelan Yang acknowledges the support by Scientific Research Fund of Hunan Provincial Education Department (Grant no. 12B023). Su-Qun Cao acknowledges the support by the National Spark Plan of China (Grant no. 2013GA690404).

Gelan Yang

Su-Qun Cao

Yue Wu 


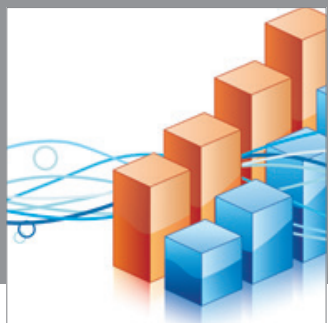

Advances in

Operations Research

mansans

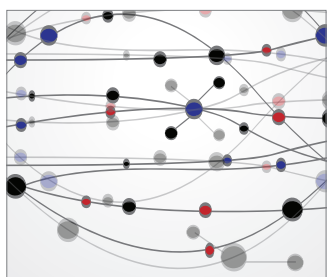

The Scientific World Journal
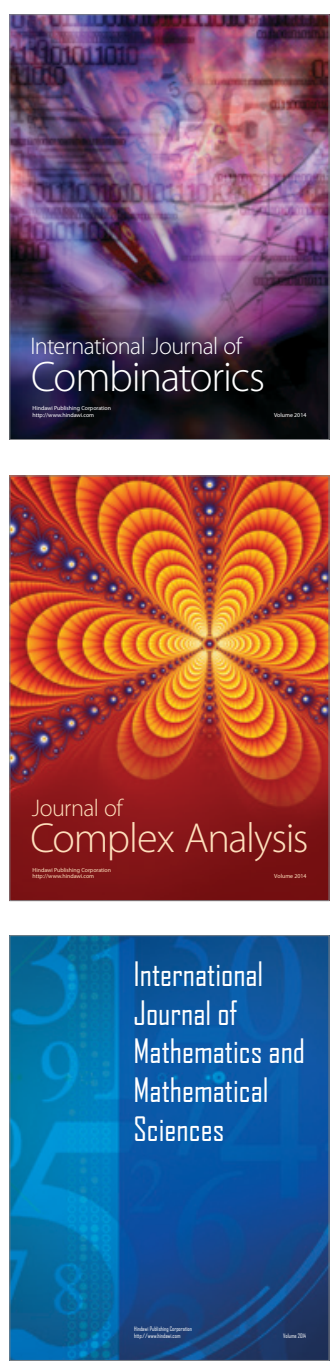
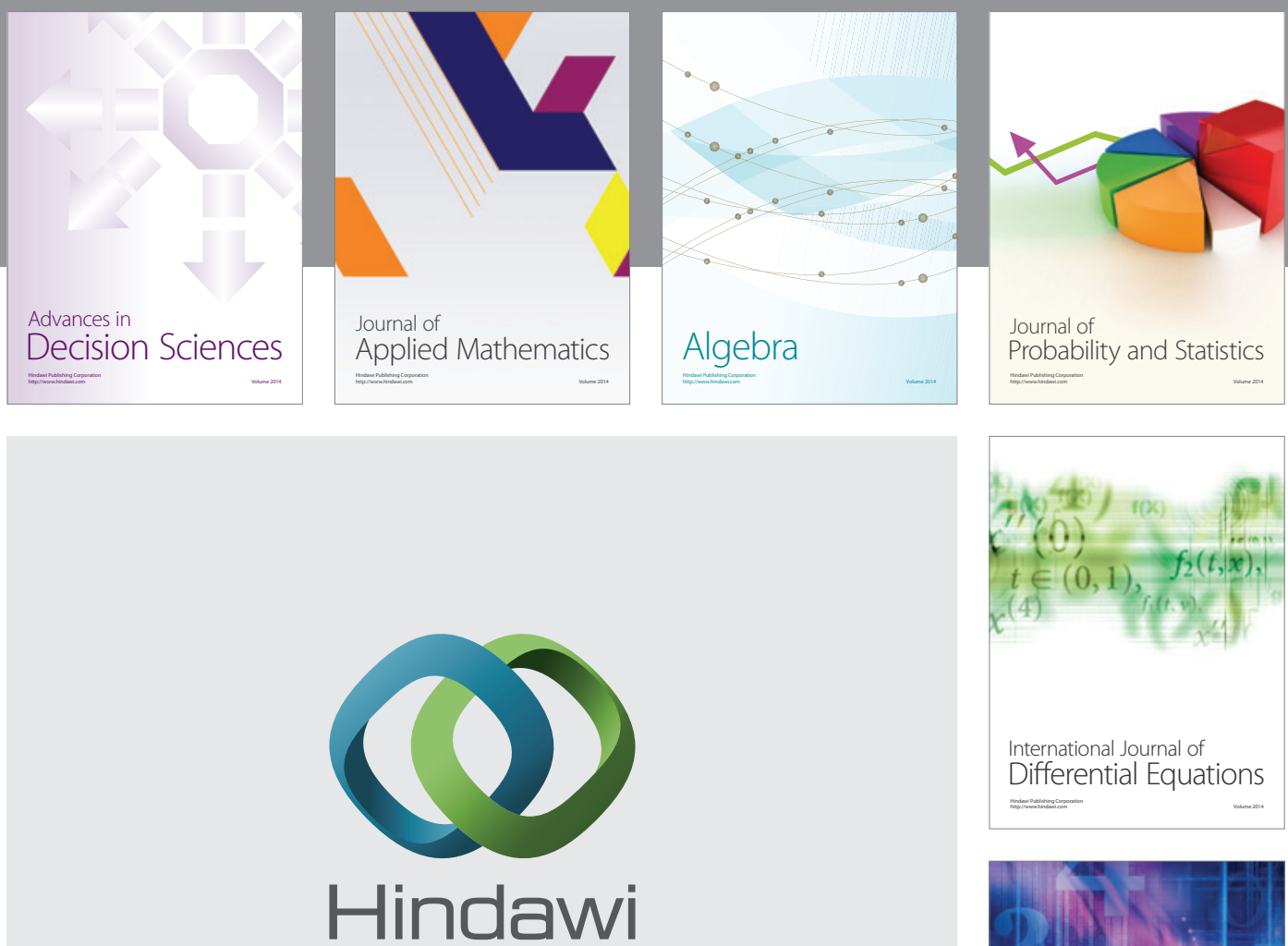

Submit your manuscripts at http://www.hindawi.com
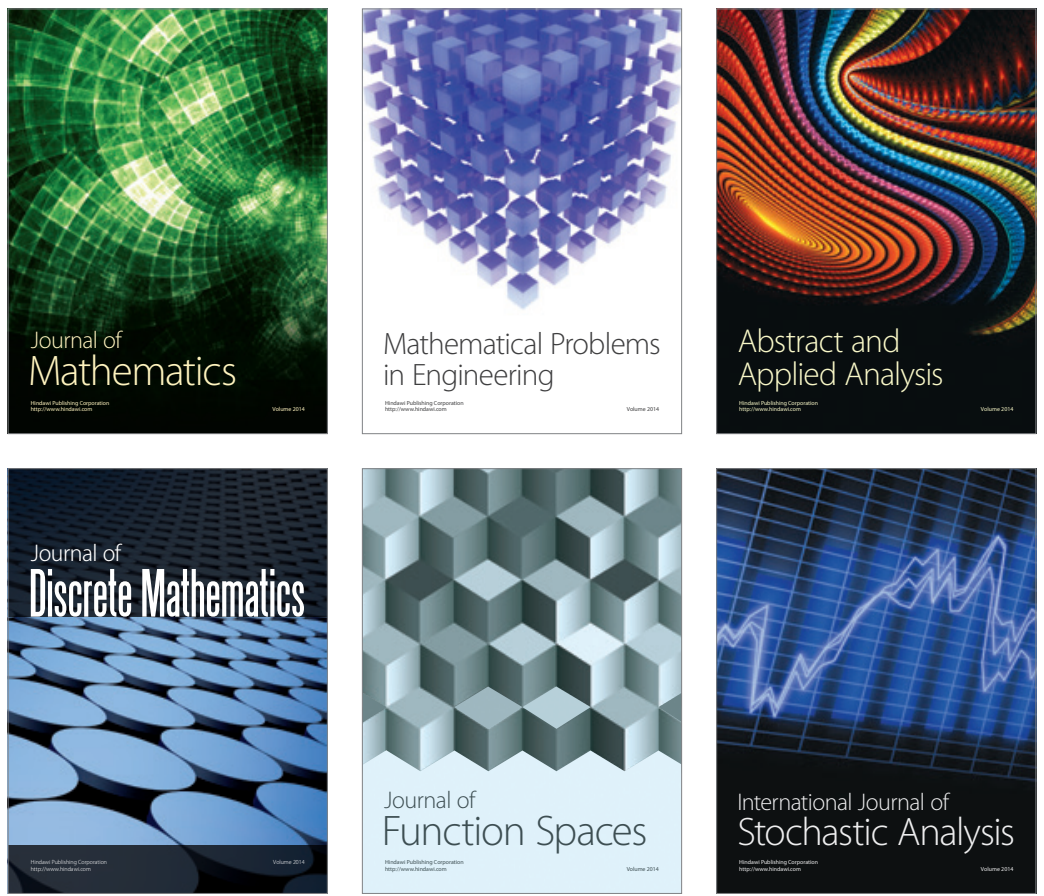

Journal of

Function Spaces

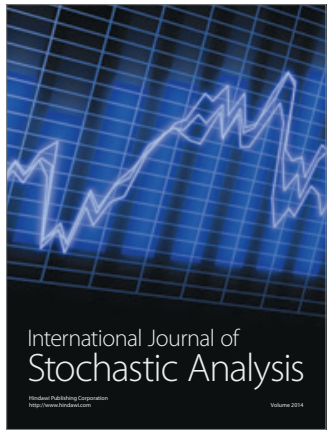

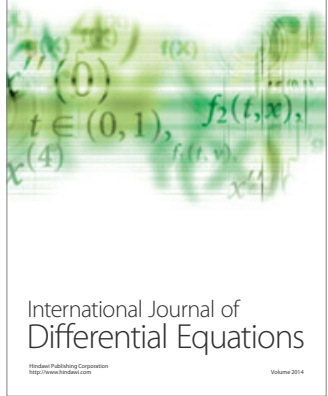
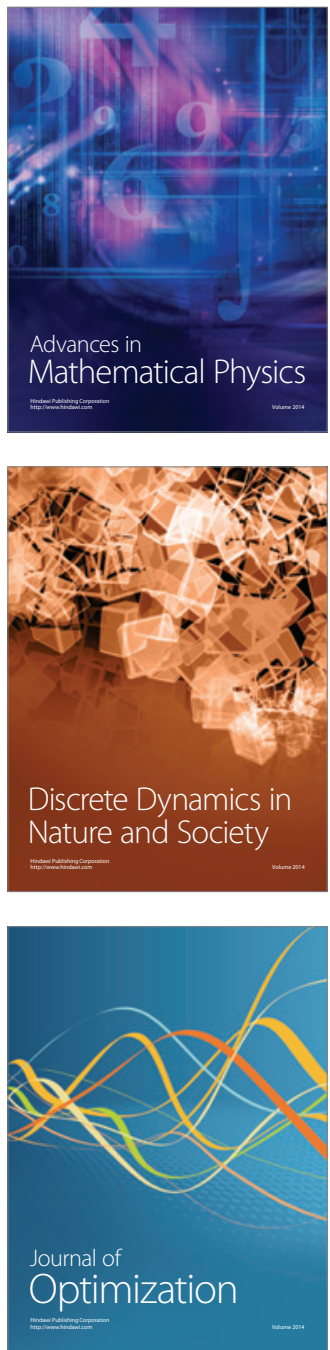